\title{
Is there a role for postinduction positron emission tomography and computed tomography with fludeoxyglucose $F$ 18? A call for standardization
}

\author{
Katie S. Nason, MD, MPH
}

\footnotetext{
From the Division of Thoracic and Foregut, Department of Cardiothoracic Surgery, University of Pittsburgh, Pittsburgh, Pa.

Supported by award K07CA151613 from the National Cancer Institute. The content is solely the responsibility of the author and does not necessarily represent the official views of the National Cancer Institute or the National Institutes of Health.

Disclosures: Author has nothing to disclose with regard to commercial support.

Received for publication Jan 15, 2016; accepted for publication Jan 15, 2016; available ahead of print Feb 9, 2016.

Address for reprints: Katie S. Nason, MD, MPH, Division of Thoracic and Foregut, Department of Cardiothoracic Surgery, University of Pittsburgh, 5200 Centre Ave, Suite 715, Shadyside Medical Building, Pittsburgh, PA 15232 (E-mail: nasonks@upmc.edu).

J Thorac Cardiovasc Surg 2016;151:980-1

$0022-5223 / \$ 36.00$

Copyright $(2016$ by The American Association for Thoracic Surgery

http://dx.doi.org/10.1016/j.jtcvs.2016.01.025
}

In their article in this issue of the Journal, "Postinduction Positron Emission Tomography Assessment of N2 Nodes Is Not Associated With ypN2 Disease or Overall Survival in Stage IIIA Non-Small Cell Lung Cancer,' Ripley and colleagues $^{1}$ sought to determine whether postinduction positron emission tomography (PET) fludeoxyglucose $\mathrm{F}$ 18 (INN fludeoxyglucose [18F]; FDG) avidity in N2 nodes was associated with persistent $\mathrm{N} 2$ nodal metastasis in patients with confirmed stage IIIA non-small cell lung cancer. The article also examined whether persistent pathologic N2 node positivity was associated with worse overall survival among patients who underwent resection. Persistent N2 node involvement was identified in $61 \%$ of patients. When N2 nodes were FDG avid on postinduction PET with computed tomography (CT), pathologic nodal involvement was confirmed in only $64 \%$ of patients; accuracy, sensitivity, and specificity were $57 \%, 59 \%$, and $55 \%$, respectively. Median survival was 3.7 years with persistent $\mathrm{N} 2$ disease, compared with 4.5 years when N2 status was negative at resection. Ripley and colleagues ${ }^{1}$ concluded that N2 nodal station FDG-PET activity was not associated with pathologic N2 nodal status, consistent with the published literature. ${ }^{2}$ In addition, they concluded that persistent nodal disease is not associated with overall survival.

The study of Ripley and colleagues ${ }^{1}$ has several strengths and weaknesses that warrant further discussion. Strengths include the histologic confirmation of N2 nodal metastasis before induction therapy and that most patients underwent postinduction PET/CT scans, allowing estimation of postinduction PET/CT accuracy for $\mathrm{N} 2$ node status. Unfortunately, secondary study aims were less well executed, leading to overstatements regarding overall survival as the study became underpowered, in large part as a result of small numbers and high rates of censoring

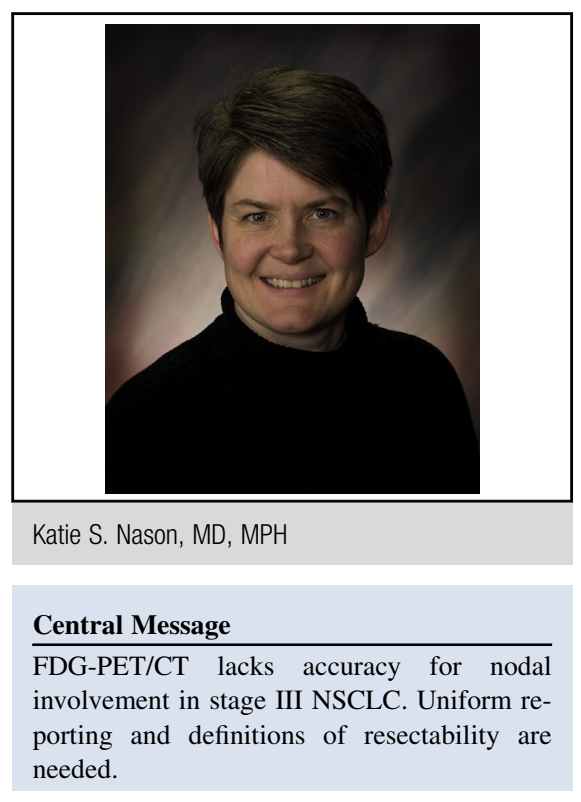

See Article page 969

See Editorial page 911.

during follow-up (eg, only $63 \%$ and $11 \%$ cases were analyzable at 2 and 5 years). The need for caution is exemplified by the lack of statistically significant increased hazard of death despite persistent disease after resection (including 2 patients with metastatic disease). In addition, univariate hazard ratio favored reduced hazard of death for pathologically persistent $\mathrm{N} 2$ disease (hazard ratio, $0.98 ; P$ not statistically significant), whereas one would expect the hazard ratio to be on the side of detrimental in this setting.

The study of Ripley and colleagues ${ }^{1}$ is provocative in the sense that it highlights important limitations in the guidelines for management of patients with N2 node positivity in non-small cell lung cancer. For future studies and to guide consistent clinical care, an objective and generalizable definition of $\mathrm{N} 2$ node resection and resectability is needed. As in this study, however, resectability is more often a subjective assessment that is based on the surgeon's determination that "they can get it all." Findings of discrete versus diffusely infiltrative nodal involvement, size criteria, or number of involved N2 nodal stations very likely influenced the surgeon's assessment of 
resectability, but lack of standard definitions and reporting make this information inaccessible and unanalyzable. Future studies that include a more objective description of resectable $\mathrm{N} 2$ disease will be extremely helpful in guiding the reader in decisions regarding resectability in their own patients and regarding limiting nontherapeutic surgical intervention.

The study of Ripley and colleagues ${ }^{1}$ also highlights limitations derived from the lack of standardization in performance and reporting of FDG-PET/CT. In this study, semiquantitative estimates of FDG avidity (maximum standardized uptake value [SUV]) were not analyzed because reporting from the various institutions was tremendously variable. Unfortunately, parameter guidelines from the American College of Radiology state that the reporting of SUV as an absolute value is optional, despite recognition in the published parameter that changes in SUV with time or therapy may be of value. According to the parameter, estimates of FDG avidity can be reported as the maximum SUV or by describing intensity of uptake relative to normal hepatic parenchymal uptake (mild, moderate, or intense). ${ }^{3}$ This lack of standardization will need to be addressed if we hope to fully capitalize on available imaging modalities guiding our treatment decisions. Importantly, a recent study by Schmidt-Hansen and associates ${ }^{4}$ showed that the sensitivity and specificity of FDG-PET/CT vary with the type of scanner, the dose of FDG, the location of the scan, and the histologic type of the tumor and should not be used alone to make decisions about resectability. Understanding these limitations is critical for both surgeons and oncologists, because these findings reinforce the need for histologic assessment of the mediastinal lymph nodes for the final determination of treatment strategy.

In summary, Ripley and colleagues ${ }^{1}$ have provided a thought provoking and potentially controversial study on the role of FDG-PET/CT in determining pathologic node status after induction in patients with stage III non-small cell lung cancer. Given the small numbers of patients in the study, caution should be exercised in using the data regarding survival in counseling patients. Surgeons and oncologists should insist that radiologists report objective measures of FDG avidity. Finally, thoracic surgeons should develop guidelines for comprehensive mediastinal staging and definitions of resectability to improve consistency in the management of stage IIIa non-small cell lung cancer and to facilitate comparisons of outcomes across centers and across nations.

\section{References}

1. Ripley RT, Suzuki K, Tan KS, Adusumilli PS, Huang J, Park BJ, et al Postinduction positron emission tomography assessment of $\mathrm{N} 2$ nodes is not associated with ypN2 disease or overall survival in stage IIIA non-small cell lung cancer. J Thorac Cardiovasc Surg. 2016;151:969-79.

2. de Cabanyes Candela S, Detterbeck FC. A systematic review of restaging after induction therapy for stage IIIa lung cancer: prediction of pathologic stage. J Thorac Oncol. 2010;5:389-98.

3. American College of Radiology. ACR-SPR practice parameter for performing FDG-PET/CT in oncology. Res. 24 - 2012 amended 2014 (Res. 39). Available at: http://www.acr.org/ /media/71B746780F934F6D8A1BA5CCA5167EDB. pdf. Accessed January 15, 2016

4. Schmidt-Hansen M, Baldwin DR, Zamora J. FDG-PET/CT imaging for mediastinal staging in patients with potentially resectable non-small cell lung cancer. JAMA. 2015;313:1465-6. 